\title{
Evoked Response Audiometry
}

National Cancer Institute

\section{Source}

National Cancer Institute. Evoked Response Audiometry. NCI Thesaurus. Code C85857.

An auditory test utilizing the electrical activity of neural impulses to localize the exact site of a pathologic process that causes the hearing loss. 